\title{
Thiourethane-functionalized fillers: biological properties and degradation resistance
}

\author{
Larissa Maria CAVALCANTE ${ }^{(a, c)}$ \\ Amanda Barreto RAMOS(a) \\ Daniela Costa SILVA ${ }^{(b)}$ \\ Gutemberg Gomes ALVES(b) \\ Karinne Bueno ANTUNES(c) \\ Carmem Silvia PFEIFER(d) \\ Luis Felipe Jochims SCHNEIDER ${ }^{(a, c)}$ \\ (a) Universidade Federal Fluminense - UFF, \\ School of Dentistry, Niterói, RJ, Brazil. \\ (b) Universidade Federal Fluminense - UFF, \\ Institute of Biology, Department of Cellular \\ and Molecular Biology, Niterói, RJ, Brazil. \\ (c) Universidade Veiga de Almeida - UVA, \\ School of Dentistry, Nucleus for Dental \\ Biomaterials Research, Rio de Janeiro, \\ RJ, Brazil. \\ (d) Oregon Health and Science University, \\ Biomaterials and Biomechanics, Portland, \\ OR, USA.
}

Declaration of Interests: The authors certify that they have no commercial or associative interest that represents a conflict of interest in connection with the manuscript.

\section{Corresponding Author:}

Larissa Maria Cavalcante

E-mail: lara_cavalcante@yahoo.com.br

htps://doi.org/10.1590/1807-3107bor-2021.vol35.0018

Submitted: April 14, 2020

Accepted for publication: June 30, 2020

Last revision: September 28, 2020
Abstract: This study determined the effect of thiourethanefunctionalized fillers (TU) on the antimicrobial properties, cytotoxicity, degree of conversion (DC), water sorption (Wsp) and solubility (Wsl) of experimental composites. TU-modified fillers were added at different ratios in experimental composites: 0 (Control-TU0), 25\% (TU25), 50\% (TU50), 75\% (TU75) and 100wt\% (TU100). The antimicrobial properties were detected through the exhaustion test and counting of Streptococus mutans colonies for biofilm formation. Cytotoxicity to human gingival fibroblasts was evaluated in three different parameters: XTT (2,3-Bis(2-Methoxy-4-Nitro-5-Sulfophenyl)-2H-Tetrazolium-5-Carboxanilide), NRU (Neutral Red Uptake assay) and CVDE (Crystal Violet Dye Exclusion test)) at the same cells. ELISA was used to measure the IL-6 and b-FGF biomarkers. DC was determined by Fourier-transformed infrared spectroscopy, while Wsp and Wsl by mass variations. Inhibitory capacity of biofilm formation was not observed for any material. All groups presented at least $70 \%$ of cell survival within the observed periods (24h and 7 days). Positive control (toxic) had high IL-6 values and low b-FGF values. No significant variations in DC, Wsp, and Wsl were observed among the experimental groups. The use of thiourethane did not present antimicrobial and cytotoxic activity and the tested materials presented equivalent properties to those conventionally used in dentistry.

Keywords: Materials Testing; Biomarkers; Anti-Infective Agents; Anti-Bacterial Agents.

\section{Introduction}

Composite resins are the main choice for a variety of clinical situations. ${ }^{1}$ Since their introduction on the market, significant changes have been made in the composition to overcome limitations and achieve improved physical, mechanical and aesthetic properties. ${ }^{2}$ In this context, thiourethane oligomers have been incorporated as an alternative to reduce the stress development and to improve materials' mechanical properties. ${ }^{3}$

Proposed as an additive to the methacrylate matrices, thio-urethanes (TU) are pre-polymerized oligomers that provide advantages to the polymerization mechanism of composite materials. ${ }^{3}$ The oligomer has pendant thiol functionalities, which react by chain-transfer with the 
methacrylate group resulting in delayed gelation and vitrification of the forming polymer network, meaning that the stiffening of the network is initiated at higher conversions. ${ }^{3,4}$ Consequently, the overall conversion is increased and the polymerization stress reduced. ${ }^{4}$ In addition, the high molecular weight of the oligomer is associated with reduction in volumetric shrinkage and production of more homogeneous networks. ${ }^{4}$ It is important to highlight that all improvements provided by the addition of TU do not require modifications of the traditional clinical restorative procedures. ${ }^{5}$

One common concern associated with thiolcontaining materials, especially in combination with methacrylates, is the potential for Michael addition reactions that reduce the shelf-life of the fully formulated materials. ${ }^{6}$ Another potential drawback with any high molecular weight additive is the potential for increased viscosity. One new approach to overcome this issue is to use thiourethanefunctionalized fillers as a coupling agent. ${ }^{7}$ By adding the methoxy-silane-modified TU to the filler surface via standard silanization methods, ${ }^{7}$ previous studies have demonstrated to be possible to still harness the same benefits observed when the TU was added to the matrix (i.e., stress reduction and increased fracture toughness), but at a much lower concentration and with much less significant impact to handling properties. ${ }^{7,8}$

In addition, failures at the interfacial bonding between inorganic fillers and organic matrix can be expected in a composite material, leading to water accumulation and compromising hydrolytic stability. As a consequence, faster degradation of the material can be observed ${ }^{9}$ and sorption and solubility properties should be investigated when particles coverage is proposed. In addition, interface failures between the inorganic filler and organic matrix may also lead to dissolution and leaching of components, such as unreacted residual monomers or filler particles. ${ }^{6}$ The compounds normally released from dental composites and adhesives are known to affect and produce cytotoxic reactions to oral cells. ${ }^{9}$ So, an analysis of the cytotoxicity of the material can be used to investigate this process and predicting the behavior of thiourethane incorporation. In addition, there is some evidence for antimicrobial activity in thiol-containing compounds $\mathrm{s}^{10}$ but such important biological aspects have not been investigated for thiourethane-containing restorative dental materials.

Due to the lack of the aforementioned information the aim of this study was to determine the effect of systematic addition of thiourethane-functionalized fillers to degree of conversion, water sorption and solubility, antimicrobial properties and the cytotoxic capacity were also evaluated. The hypotheses tested were that the use of thiourethane-functionalized fillers would a) increase the degree of conversion - being directly proportional to the concentration of thio-urethane functionalized fillers used in the formulation, $b$ ) reduce the water absorption and solubility, c) do not provide antimicrobial properties and d) no cytotoxic effects.

\section{Methodology}

\section{Model composites formulation}

The mixture Bis-phenol A diglycidyl dimethacrylate (Bis-GMA), urethane dimethacrylate (UDMA) and tri-ethylene glycol dimethacrylate (TEGDMA), was used at 50:30:20 mass ratio (all monomers from Esstech Inc., Essington, USA). To this, 0.2\% camphorquinone (Esstech Inc., Essington, USA) and 0.6\% EDMAB amine (Sigma-Aldrich, Milwaukee, USA) were added as the photoinitiator/co-initiator system. Fillers were added at $70 \%$ mass, with $90 \%$ composed by borosilicate barium glass silane-treated particles (0.7 $\mu \mathrm{m}$ average size, Esstech Inc., Essington, USA) functionalized or not with thiourethane and 10\% of spherical silica MPTS-silane-treated particles (Aerosil OX50, Evonik Degussa, Essen, Germany). Table 1 provides a systematic explanation regarding the filler content of each formulation.

The thiourethane functionalization procedures were described in detail in a separate publication. ${ }^{7}$ In brief, the thiourethane oligomers were synthesized in solution, by combining 1-isocyanato-4-[(4isocyanatocyclohexyl) methyl] cyclohexane (DHDI) with trimethylol-tris-3-mercaptopropionate (TMP), purified by precipitation in hexanes. The oligomer was then grafted to the surface of the filler particles using standard protocols ${ }^{11}$ in an acidified ethanol solution, followed by ball-milling and drying. The 
Table 1. Composite formulation and experimental groups definition .

\begin{tabular}{|c|c|c|}
\hline $30 \%$ organic matrix & \multicolumn{2}{|c|}{$70 \%$ inorganic filler } \\
\hline BisGMA/UDMA/TEGDMA & 10\% OX-50: $0.04 \mu \mathrm{m}$ & Groups \\
\hline \multirow{5}{*}{ 50:30:20 mass ratio } & \multirow{5}{*}{$90 \%$ Barium Glass: $0.7 \mu \mathrm{m}$} & TU0: no functionalized filler \\
\hline & & TU25: $75 \%$ without TU $+25 \%$ with TU \\
\hline & & TU50: $50 \%$ without TU $+50 \%$ with TU \\
\hline & & TU75: $25 \%$ without TU $+75 \%$ with TU \\
\hline & & TU100: 100\% with TU functionalized filler \\
\hline
\end{tabular}

particles were characterized by thermogravimetrical analysis to ensure silanization, which accounted for $8 \%$ of the mass loss. Thiourethane-functionalized fillers were systematically added according to the experimental groups detailed in Table 1. Thiourethanefunctionalized fillers were systematically added according to the experimental groups expressed in Table 1. A mechanical mixer (SpeedMixer, DAC 150.1 FVZ, FlackTek Inc., Germany) was used for $5 \mathrm{~min}$ at $2500 \mathrm{rpm}$ to produce homogeneous pastes.

All photoactivation procedures were performed with an LED-based curing unit for 40 seconds at an incident irradiance of $1100 \mathrm{~mW} / \mathrm{cm}^{2}$ (Emitter.B Supra, Schuster Dental Equipments Ltda, Santa Maria, Brazil).

\section{Degree of conversion}

DC was determined by Fourier-transformed infrared spectroscopy $(n=3)$, using a spectrometer equipped with an attenuated total reflectance (ATR) diamond device (Brüker Optics, Ettinger, Germany). A dark circular plastic mold (diameter $7 \mathrm{~mm}$, thickness $0.3 \mathrm{~mm}$ ) was used for individually accommodation of each material over the ATR crystal. A Mylar strip was placed on the surface and a spectrum from the uncured material was taken in the absorbance mode using 32 scans and $4 \mathrm{~cm}^{-1}$ resolutions. Photoactivation was performed and a second spectrum was obtained after $5 \mathrm{~min}$. \%DC was calculated using baseline technique and considering the differences in intensity of $C=C$ stretching vibration (peak area) at $1638 \mathrm{~cm}^{-1}$ of uncured and cured spectra, with the symmetric aromatic stretching at $1608 \mathrm{~cm}^{-1}$ (peak area) as internal standard. The following formula was then used:

$\% \mathrm{DC}=100\left\{1-\left[\frac{\mathrm{C} \text { cured/Aromatic cured }}{\text { C uncured/Aromatic uncured }}\right]\right\}$

\section{Sorption and solubility}

The material was inserted in a single increment in a metal mold ( $\varnothing 8 \mathrm{~mm} \times 2 \mathrm{~mm}$ thickness) between Mylar strips and with a glass coverslip on top of which a weight of $500 \mathrm{~g}$ was placed $(\mathrm{n}=5)$. After photoactivation, samples were removed and stored at room temperature and protected from light. After $24 \mathrm{~h}$ both surfaces were mechanically polished with 2,000 and 4000-grit SiC papers.

The samples were then stored in a desiccator with silica gel for $22 \mathrm{~h}$, at $37 \pm 1^{\circ} \mathrm{C}$. Then samples were transferred to a desiccator at room temperature, for $2 \mathrm{~h}$. They were weighed in a $24 \mathrm{~h}$ cycle with an analytical balance, accuracy $0.01 \mathrm{mg}$, (XS105 DU, Mettler-Toledo, Leicester, UK) until obtaining a constant weight (range smaller than $\pm 0.1 \mathrm{mg}$ for three consecutive weighing - m1). The thickness and diameter of the samples were measured with a digital caliper (ABS digital caliper, Mitutoyo, Tokyo, Japan) at four equidistant points, and the volume $(\mathrm{V})$ was calculated in $\mathrm{mm}^{3}$. Afterwards, the samples were placed individually in glass phials, immersed in $10 \mathrm{ml}$ of distilled water, and weighed in a $24 \mathrm{~h}$ cycle for 30 days until reaching the equilibrium (m2). After equilibrium was reached, they were taken back to the desiccator and weighed every $24 \mathrm{~h}$, repeating the same cycles required to obtain $\mathrm{m} 1$, until the mass equilibrium (m3) was reached. Water sorption (Wsp) and solubility (Wsl), both in $\mathrm{mg} / \mathrm{mm}^{3}$, were then calculated according the two following formulas:

$$
\mathrm{Wsp}=(\mathrm{m} 2-\mathrm{m} 3) / \mathrm{V} \text { and Wsl }=(\mathrm{m} 1-\mathrm{m} 3) / \mathrm{V} \text {. }
$$

\section{Antimicrobial assay}

Composites were placed on metallic molds and covered with a polyester strip before the 
photoactivation. Six discs (Ø $4 \mathrm{~mm} \times 2 \mathrm{~mm}$ h) per group were prepared. After photoactivation, samples were removed and stored at room temperature and protected from light. In order to obtain similar surface texture, samples were polished (Aropol-e, AROTEC, Cotia, Brazil) with 600-, 800- and 1,200- grit $\mathrm{SiC}$ sandpapers at $150 \mathrm{rpm}$ for $60 \mathrm{~s}$ under continuous water-cooling.

Antimicrobial action on S. mutans ATCC 25175 was carried out through the Exhaust Test, previously reported by Perez et al., ${ }^{12}$ which has as its principle to test the samples after elution periods with sterile distilled water. The samples, divided according to the previously established groups (TU0, TU25, TU50, TU75 and TU100), were inserted into cell culture plate of 96 wells $(6.86 \varnothing)$ and were submitted to elution with daily changes of distilled and deionized water in the volume of $250 \mu 1$, performed in a laminar flow chapel. Elution times varied from 0 (without exhaustion), 15, 30 and 60 days.

S. mutans growth was conducted in triplicate, with each specimen inserted into a 96 well flat-bottomed cell culture plate (Corning, NY, USA). The inoculum was prepared from the growth of $S$. mutans ATCC 25175 in $5 \mathrm{~mL}$ of Trypticase Soy Broth (TSB, Difco, Rio de Janeiro, RJ, Brazil) for $24 \mathrm{~h}$ at $37^{\circ} \mathrm{C}$. The cells were then centrifuged and washed in phosphate buffered saline (PBS, 0.01M, pH 7.2) and suspended in PBS, in a concentration of $\mathrm{OD}=0.16 \mathrm{in} 580 \mathrm{~nm}$ wavelength, to obtain a concentration of $1 \times 108$ colony forming units per milliliter (CFU/mL). The suspension was diluted in PBS to obtain the working suspension containing approximately $3 \mathrm{CFU} / \mu \mathrm{L}$. Then, $25 \mu \mathrm{L}$ was dispensed on the surface of each specimen and kept in contact for $30 \mathrm{~min}$. After this time, $200 \mu \mathrm{l}$ of the Trypticase Soy Agar medium (TSA, Difco Labs, Detroit, USA) containing $0.01 \%$ 2,3,5-triphenyltetrazolium chloride (TTFC) was added to each well for growth and enable the visualization of the colonies. The plate was incubated at $37^{\circ} \mathrm{C}$ for $48 \mathrm{~h}$ in carbon dioxide $\left(\mathrm{CO}_{2}\right)$ presence. The method used for CFU count in the 96-well plates was performed with a binocular stereoscope. The wells were observed after 24 and 48 hours of incubation for the quantification of CFU. Colony counts in each well was expressed and compared to the total viable organisms. The antimicrobial activity was measured by differences in CFU counted in the stereoscopic microscope Nikon C-DSD 230 (Nikon Corporation, Japan).

\section{Cytotoxicity assay}

Disc specimens ( $\varnothing 5 \mathrm{~mm} \times 2 \mathrm{~mm}$ h) were sterilized with gamma radiation (STERRAD, Antônio Pedro University Hospital, Niterói, Brazil). Specimens were weighed to calculate the amount of medium to be added for the extracts' preparation.

According to ISO standards (10993-12:2012), extracts were prepared in a culture medium (high glucose DMEM) without fetal bovine serum and containing $1 \%$ penicillin-streptomycin solution in a proportion of $0.2 \mathrm{~g}$ material to be tested for each $\mathrm{ml}$ of medium. The specimens were immersed in the medium and incubated at $37^{\circ} \mathrm{C}$ and $5 \% \mathrm{CO}_{2}$ in a humidified chamber for $24 \mathrm{~h}$. In order to emulate long-term extraction of toxicants in the oral cavity, specimens of each group were also kept in the culture media for 7 days. The culture medium was renewed daily in order to simulate the effects of crevicular gingival fluid on clearing the material in the mouth. Extracts of negative control groups were prepared using polystyrene and high glucose DMEM without fetal bovine serum. For positive control group latex fragments were used on culture medium, which according to ISO-based assays ${ }^{13}$ presents consistent cytotoxicity.

Human gingival fibroblast cells were obtained from the repository at the Laboratory Experimental of cell culture of the Federal Fluminense University, and cultured in high glucose DMEM (GIBCO Invitrogen, Grand Island, USA) containing 10\% fetal bovine serum and $1 \%$ penicillin-streptomycin antimicrobial solution. Cell cultures were incubated at $37^{\circ} \mathrm{C}$ and $5 \% \mathrm{CO}_{2}$ in bottles of $25 \mathrm{~cm}^{3}$. After culture confluence, the cells were treated with trypsin, counted in Neubauer's chamber and subcultured in 96-well sterile plates at a cell density of $10^{4}$ cells per well.

The test was performed in quintuplicates. In each well were added $180 \mu 1$ of one of the extracts (as described above) and $20 \mu \mathrm{l}$ of fetal bovine serum, obtaining a final volume of $200 \mu \mathrm{l}$ of extract per well, and incubated for 24 hours at $37^{\circ} \mathrm{C}$ and $5 \%$ $\mathrm{CO} 2$. The possible cytotoxicity of the extracts was 
evaluated through a multiparametric test, which tests three different parameters sequentially in the same exposed cells.

The first parameter tested was mitochondrial activity, as measured by the XTT test, through the ability of dehydrogenase enzymes to convert the XTT reagent into orange-colored soluble compounds of formazan. Cell activity is directly linked to the color that is formed measured by their absorbance at 480nm with a microplate UV/Vis spectrophotometer (PowerWave MS2; BioTek Instruments, Rio de Janeiro, Brazil). After washing with Phosphate-Saline Buffer (PBS), the second method employed was the Neutral Red Uptake assay (NRU). This test uses a neutral red dye that is accumulated on lisosomes in living cells but leaks into cells that have ruptured membrane. The living cells are marked in red. After dye extraction, the Optical Density (O.D.) was measured at 540nm, and directly proportional to the number of cells with intact membranes. The third assay (Crystal Violet Dye Exclusion - CVDE) measures the amount of DNA present in the plaque, directly related to the total cell density. After washing for the elimination of the excess dye, the absorbance at $540 \mathrm{~nm}$ is proportional to the amount of cells in the well.

To obtain a more predictive evaluation of possible responses that these fibroblasts could have to apparently non-toxic materials, two important markers were measured: Interleukin-6 (IL-6) and Basic Fibroblastic Growth Factor (b-FGF). FGF is the main inducing fibroblasts growth factor, whose high level of release is associated to wound healing and a good response to biomaterials in healthy tissues. IL-6 is a pro-inflammatory cytokine released by many cells, including fibroblasts, which can be associated to a local inflammatory response.
The two markers were measured by Enzyme Linked Immuno Sorbent Assay (ELISA), employing commercially available Human FGF-basic and Human IL-6 ELISA Development Kits (PeproTech, New Jersey, USA), with sensitivity range of 63-4000pg/mL for FGF and $24-1500 \mathrm{pg} / \mathrm{mL}$ for IL-6. The experiment was performed in triplicates. The culture supernatants of exposed cells from the cytotoxicity assay described above were collected after 24 hours of incubation, pooled and stored at $-80^{\circ} \mathrm{C}$ until analysis. A negative control was used for determination of basal release of the cytokine and growth factor. The procedure followed the manufacturer's conditions and recommendations.

\section{Statistical Analyses}

Degree of conversion, Wsp, Wsl and antimicrobial data were evaluated for normal distribution by Anderson-Darling test and the parametric oneway analysis of variance. The results of ELISA and cytotoxicity assay were evaluated for normal distribution by the D'Agostino normality test and the nonparametric Kruskal-Wallis test. For comparisons, Dunn's post-test was employed. An alpha error of 5\% was considered for all statistical analysis.

\section{Results}

Data of degree of conversion, water sorption and solubility are shown in Table 2.

\section{Degree of conversion}

Model composites varying concentrations TU had no statistical difference in the degree of conversion and did not differ from the control group $(p=0.089)$.

Table 2. Mean of and standard deviations of degree of conversion (DC), water sorption (Wsp) and solubility (Wsl).

\begin{tabular}{lccc}
\hline Group & $\mathrm{DC}$, in $\%(\mathrm{n}=3)$ & $\mathrm{Wsp}$, in $\mu \mathrm{g} / \mathrm{mm}^{3}(\mathrm{n}=5)$ & $\mathrm{Wsl}, \mathrm{in} \mu \mathrm{g} / \mathrm{mm}^{3}(\mathrm{n}=5)$ \\
\hline TU0\% & $64.3(3.2)$ & $2.5(0.2)$ & $5.1(2.3)$ \\
TU25\% & $63.1(1.1)$ & $2.4(0.4)$ & $2.7(1.3)$ \\
TU50\% & $69.0(1.0)$ & $2.3(0.1)$ & $3.1(0.9)$ \\
TU75\% & $65.6(2.0)$ & $2.3(0.1)$ & $4.7(3.5)$ \\
TU100\% & $64.0(3.6)$ & $2.4(0.2)$ & $2.8(1.9)$ \\
\hline
\end{tabular}

p-value was 0.089 for DC; 0.720 for Wsp; and 0.265 for Wsl. No statistical differences were observed for DC, WS and SL. 


\section{Sorption and solubility}

The addition of TU coating inorganic fillers did not adversely affect water sorption and solubility. No statistically significant differences were detected among the experimental groups. $(\mathrm{p}=0.720$ and $\mathrm{p}=0.265)$.

\section{Antimicrobial assay}

No significant antimicrobial properties were observed for the experimental groups. Binocular stereoscopic microscope images evidenced bacterial growth in all experimental groups in the presence of test specimens and in the control group of the experiment without the test specimen. The technique described permitted not only the visualization of the growth of microorganisms but also the quantification of colonies forming units (CFUs) in each group (Figure 1). Although the control group presented the highest values of $\mathrm{CFU}$, no statistical significant difference was detected among the experimental groups. Thus, the addition of TU was not able to inhibit the bacterial growth.

\section{Cytotoxicity assay}

Figure 2 shows the results of the multiparametric test of cytotoxicity by indirect exposure (to extracts) according to ISO 7405:2018. ${ }^{14}$ None of the experimental groups presented a different behavior between $24 \mathrm{~h}$ and

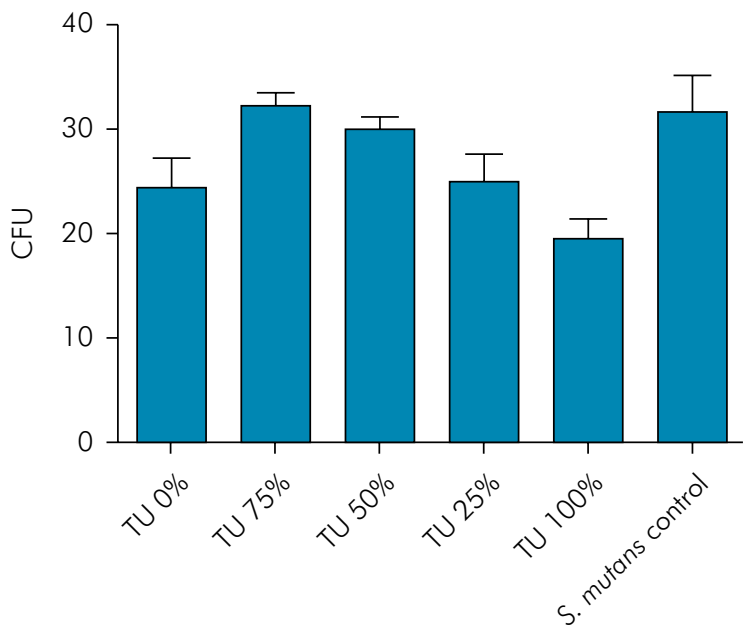

Figure 1. Colonies forming units (CFUs) in each experimental group and control group.
7 days of exposure. In both cases, no tested material presented levels below $70 \%$ of cellular survival when compared to control (Figure 2A, 2B and 2C), while
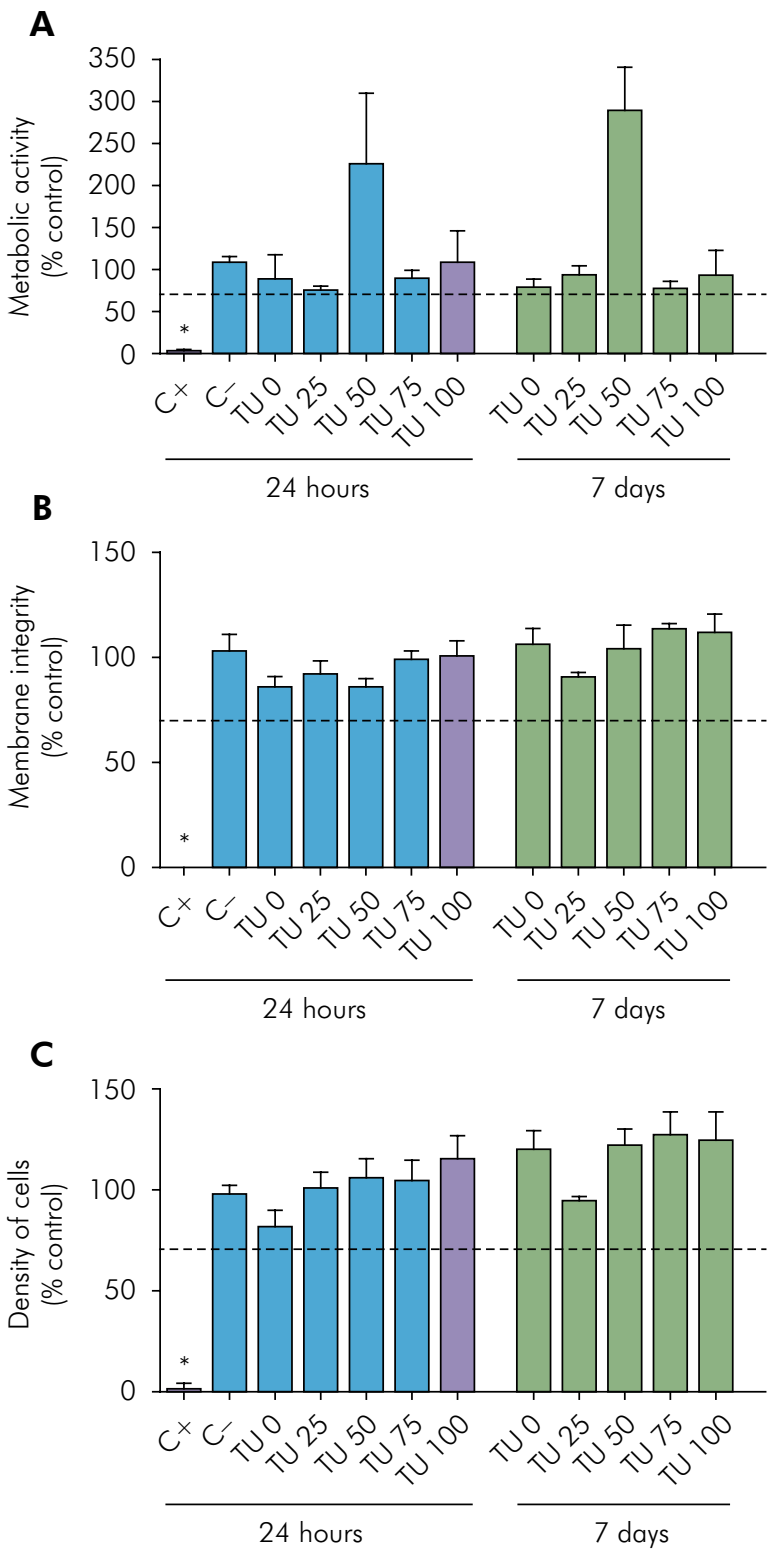

Figure 2. Multiparametric Cytotoxicity Assay. HGF cells were exposed to extracts produced by incubation for 24h (black bars) or 7 days (white bars) of each material, or to a positive control (Latex, C+), or a negative control (Polystyrene beads, C-). Cell survival was measured by the XTT (A), NRU (B), or CVDE (C) assays. Results represented as mean \pm SD of quintuplicates, as a percentage of an unexposed group (Control). The dashed line identifies the cutoff of $70 \%$ cell survival for non-cytotoxic materials, according to ISO 7405:2008. The asterisk indicates significant difference from all other groups $(p<0.05)$. 
A

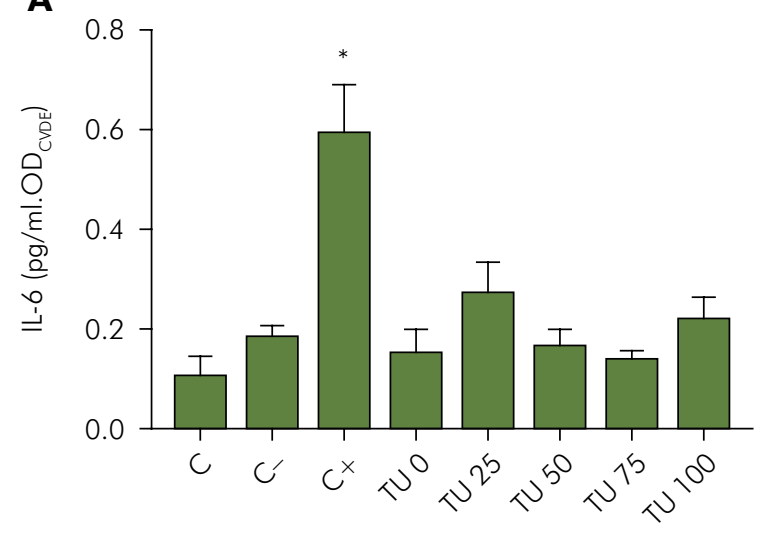

B

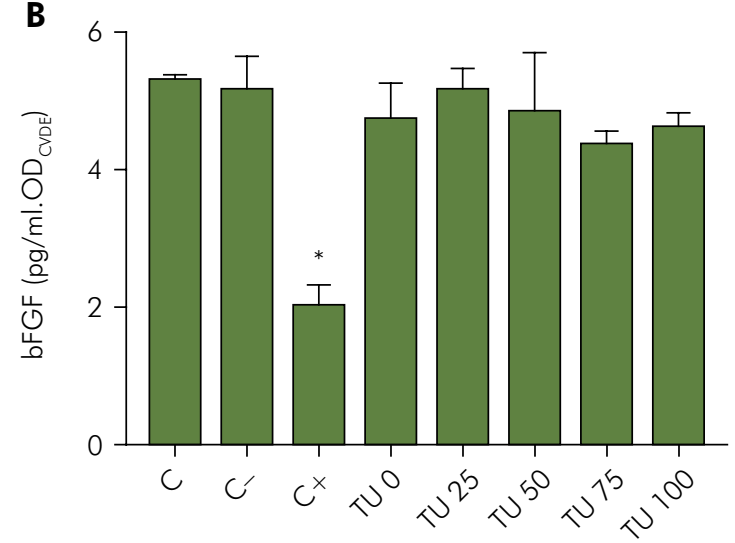

Figure 3. Evaluation of the release of IL-6 (A) and b-FGF (B) by HGF cells exposed to 24 hours extracts of the tested materials, or to a positive control (Latex, C+), or to a negative control (Polystyrene beads, C-). The results from the ELISA assay for cytokines (pg/ $\mathrm{ml}$ ) was normalized by the cell density obtained with a CVDE assay performed in the same cells. The asterisk indicates significant difference from all other groups $(p<0.05)$.

the positive control (latex) was significantly cytotoxic $(p<0.05)$. In the graph, it can be seen that the groups tested had similar results to the negative control. Thus, no group showed cytotoxicity by the three parameters evaluated (metabolic activity, integrity and cell density) regardless of time (24h or 7 days). However, an increased metabolic activity $(p<0.05)$ was observed in cells exposed to TU 50, in both experimental times.

The results indicated that the biocompatibility of the tested materials extends to the cellular response to the two markers: IL-6 and b-FGF (Figure 3). Figure 3A shows that the cytotoxic positive control (latex) killed the cells during $24 \mathrm{~h}$ and induced these to a strong release of IL-6, while all the tested materials had similar results to the negative control. Figure 3B shows the cell response to b-FGF, in which it is possible to observe once again an impact of the exposure to the positive control, this time reducing the release of growth factor, while all other groups maintained similar responses to the control.

\section{Discussion}

Improved conversion was expected as previous studies have frequently found higher DC when TU is added to the material's formulation., ${ }^{4,5,15}$ However, the addition of thiourethane-treated fillers did not statistically affect the DC in the current study and the first research hypothesis was rejected.

This result is not entirely surprising, since in the aforementioned studies the TU oligormer was added directly to the resinous matrix at a higher overall concentration - $6 \mathrm{wt} \%$ of the overall material mass, whereas in the present study, the overall concentration of TU was in the range between 1.2-4.8 wt $\%$ (detailed calculations shown in Faria-e-Silva $(2019)^{8}$. The authors of those studies hypothesized that the reduction in stress and increase in toughness was brought by the concentration of the stress-relieving oligomer at the particle-matrix interface, ${ }^{7,8,16}$ where a large portion of the stresses is thought to be concentrated. However, for the delayed gelation/vitrification to ultimately lead to improved overall conversion, it was theorized that the TU would have to be localized within the matrix. ${ }^{716}$ This was also observed in this study, as the conversion was not affected by the addition of the TU as a particle surface treatment. The current study fills a gap in the TU literature and mainly concentrates in determining the biological properties and the degradation resistance. Therefore, the fact that the DC was not negatively affected is considered positive.

The second research hypotheses was rejected as water sorption $(p=0.720)$ and solubility $(p=0.265)$ values were equivalent to control. This behavior was also found in previous studies that used TU 
precursor compounds such as thiol-ene-methacrylate ${ }^{16}$ and urethane-based multifunctional methacrylate monomer. ${ }^{17}$ Boulden et al. ${ }^{18}$ showed that water sorption of methacrylate-thiol-ene had a significant decrease when compared to control and solubility was equivalent but also without statistical difference.

The third research hypothesis was accepted as TU-based materials did not present antimicrobial properties. Antimicrobial materials have been developed for the purpose of killing the bacteria by contact (bactericidal effect) or preventing bacterial adherence (anti-biofilm effect). ${ }^{3}$ The researches has focused on the addition of this property to the restorative materials since the main reasons for the failure in composite resin restoration are the development of secondary caries due to the formation of biofilms in the gaps of the restoration margins. ${ }^{19,20,21}$ Thus, all new material introduced should seek to evaluate the presence of such antimicrobial activity.

Being Streptococcus mutans the main etiologic agent of caries disease, this microorganism was used in this assay. It is very important to identify specifically how sensitive this specie was to the experimental composites containing thiourethane-functionalized fillers. The technique recommended in this study to evaluate the antimicrobial properties has already been used successfully in previous study. ${ }^{12}$ This technique permitted not only the visualization of the growth of microorganisms but also the quantification of colonies forming units (CFUs) in each group.

Images from binocular stereoscopic microscope showed that $S$. mutans colonies were formed in all wells that had the presence of the experimental material at baseline and after 60 days exhaustion, a period in which it was believed that the specimens could release compounds in the medium that had antimicrobial action. This period was based on the time of absorption and solubility that TU presented in the tests of this work. However, even after this period no significant antimicrobial properties were observed. Such a property was unknown in materials using TU, since no study had yet appraised that issue.

The evaluation of cytotoxicity was done through a multiparametric test, which assesses three different parameters using the same cells. This allows a greater understanding of the cytotoxicity phenomenon and a greater chance of detecting changes that only one test would not detect. In this study, such methodology identified a curious effect for the TU 50 group, which has shown a threefold increase in the metabolic activity, but in the other two tests presented a compatible result with the other groups tested. This fact indicates that there was an increase in metabolism, however, without changes in cell survival or integrity. This result reaffirms the importance of the use of diverse parameters for the analysis of cytotoxicity.

While the present results could not provide an explanation for this increased metabolic activity independent of time (24h or 7 days) in the TU 50 group, two hypotheses can be considered. A first hypothesis would be the possible interference of some reducing component in the material, converting XTT into formazan independent of the presence of cells. This hypothesis, however, is discarded by a test where it is possible to verify that this material does not have the capacity to cause this interference in the absence of cells (data not shown). Another hypothesis would be that the material is causing a disturbance that generates an exacerbated response in the cells. This may be associated to an oxidative stress conditions. However, this study did not advance to prove this hypothesis, especially considering that the material of this group did not present prominence in the other parameters evaluated. Although the TU50 group was not cytotoxic, it was not considered as optimal for this stage of the biological evaluation.

It is known that dental materials release substances in different degrees in the buccal environment. ${ }^{22}$ Cells present in such medium can react with such components releasing mediators that can promote proliferation and differentiation of tissues culminating in a positive response to the material as well as the release of stress markers which may, for example, exacerbate a local inflammatory response. The cells selected in this study (HGF) are important representatives for the in vitro evaluation of dental materials, allowing the investigation of the release of growth factors, such as b-FGF, and cytokines, such as IL-6, that may affect biocompatibility. ${ }^{23}$ The results of the present study indicate that the biocompatibility of the tested materials extends to the response of the markers, where it was possible 
to observe that the cytotoxic positive control (latex), besides killing the cells during 24 hours, induced them to a strong liberation of IL- 6, which did not occur in the experimental groups. The IL- 6 marker is associated with a representative stress and immune response. ${ }^{24}$ Regarding b-FGF, it was possible to observe that the experimental groups had results equivalent to C-, which means that they are not inducing a cellular proliferation, and also not killing. It is important to highlight that the results of TU50 group is corresponding to the other experimental groups an C-, and the stress that is being produced in those cells that are breathing more, is not causing more release of pro-inflammatory cytokines when compared to the other groups. The TU zero, which would be the commercial standard, does not present much better results than the experimental groups. The fourth research hypothesis was then accepted, as TU-based materials were not cytotoxic.

The current study is the first to determine the effect of thiourethane-functionalized fillers on the antimicrobial properties and cytotoxicity of dental composites, which were not affected. These parameters are usually related to the concentration applied from a given component and is important to highlight that the present outcomes are limited to the amount of thiourethane-functionalized fillers evaluated. Therefore, further studies are still necessary to systematic evaluate if higher concentrations could affect the biological properties and the degradation resistance of the final material.

\section{Conclusion}

Based on the results of this study, it can be concluded that the addition of thiourethane-functionalized fillers to methacrylate-based experimental composites maintained the sorption and solubility standards equivalent to conventional materials, as well as the degree of conversion. The material did not present antimicrobial properties neither cytotoxic activity to the cells.

\section{Acknowledgments}

The authors dedicate this article to the memory of Prof. Dr. Raphael Hirata Junior, from the School of Medical Sciences (UERJ), who performed the antimicrobial assay. This article involves part of the results from the $\mathrm{AB} \mathrm{PhD}$ thesis. LMC and LFS are grateful to Funadesp for research scholarships. LFS is grateful to Faperj for research scholarship (JCNE E-26/202.735/2018).

\section{References}

1. Ferracane JL. Resin composite: state of the art. Dent Mater. 2011 Jan;27(1):29-38. https://doi.org/10.1016/i.dental.2010.10.020

2. Cramer NB, Stansbury JW, Bowman CN. Recent advances and developments in composite dental restorative materials. J Dent Res. 2011 Apr;90(4):402-16. https://doi.org/10.1177/0022034510381263

3. Fugolin AP, Pfeifer CS. New resins for dental composites. J Dent Res. 2017 Sep;96(10):1085-91. https://doi.org/10.1177/0022034517720658

4. Bacchi A, Consani RL, Martim GC, Pfeifer CS. Thio-urethane oligomers improve the properties of light-cured resin cements. Dent Mater. 2015 May;31(5):565-74. https://doi.org/10.1016/i.dental.2015.02.008

5. Bacchi A, Pfeifer CS. Rheological and mechanical properties and interfacial stress development of composite cements modified with thio-urethane oligomers. Dent Mater. 2016 Aug;32(8):978-86. https://doi.org/10.1016/j.dental.2016.05.003

6. Elshereksi NW, Ghazali M, Muchtar A, Azhari CH. Review of titanate coupling agents and their application for dental composite fabrication. Dent Mater J. 2017 Sep;36(5):539-52. https://doi.org/10.4012/dmi.2016-014

7. Faria-E-Silva AL, Dos Santos A, Tang A, Girotto EM, Pfeifer CS. Effect of thiourethane filler surface functionalization on stress, conversion and mechanical properties of restorative dental composites. Dent Mater. 2018 Sep;34(9):1351-8. https://doi.org/10.1016/i.dental.2018.06.023

8. Faria-E-Silva AL, Dos Santos A, Girotto EM, Pfeifer CS. Impact of thiourethane filler surface functionalization on composite properties. J Appl Polym Sci. 2019 Jul;136(25):47687. https://doi.org/10.1002/app.47687

9. Gupta SK, Saxena P, Pant VA, Pant AB. Release and toxicity of dental resin composite. Toxicol Int. 2012 Sep;19(3):225-34. https://doi.org/10.4103/0971-6580.103652 
Thiourethane-functionalized fillers: biological properties and degradation resistance

10. Ghannoum MA, Eweiss NF, Bahajaj AA, Qureshi MA. Antimicrobial activity of some thiol-containing heterocycles. Microbios. 1983;37(149-150):151-9.

11. Matinlinna JP, Lassila LV, Vallittu PK. The effect of five silane coupling agents on the bond strength of a luting cement to a silica-coated titanium. Dent Mater. 2007 Sep;23(9):1173-80. https://doi.org/10.1016/i.dental.2006.06.052

12. Perez CR, Hirata Junior R, Sérgio PP. Evaluation of antimicrobial activity of fluoride-releasing dental materials using a new in vitro method. Quintessence Int. 2003 Jun;34(6):473-7.

13. International Standard Organization - ISO. ISO 10993-12:2012 Biological evaluation of medical devices — Part 12: Sample preparation and reference materials. Geneva: International Standard Organization; 2012.

14. International Standard Organization - ISO. ISO 7405:2018 Dentistry - Evaluation of biocompatibility of medical devices used in dentistry. Geneva: International Standard Organization; 2018.

15. Bacchi A, Nelson M, Pfeifer CS. Characterization of methacrylate-based composites containing thio-urethane oligomers. Dent Mater. 2016 Feb;32(2):233-9. https://doi.org/10.1016/i.dental.2015.11.022

16. Fugolin AP, Costa AR, Kono E, Quirk E, Ferracane JL, Pfeifer CS. Influence of the organic matrix composition on the polymerization behavior and bulk properties of resin composites containing thiourethane-functionalized fillers. Eur Polym J. 2020 May;130:130. https://doi.org/10.1016/i.eurpolymi.2020.109664

17. Park JG, Ye Q, Topp EM, Misra A, Spencer P. Water sorption and dynamic mechanical properties of dentin adhesives with a urethane-based multifunctional methacrylate monomer. Dent Mater. 2009 Dec;25(12):1569-75. https://doi.org/10.1016/j.dental.2009.07.010

18. Boulden JE, Cramer NB, Schreck KM, Couch CL, Bracho-Troconis C, Stansbury JW, et al. Thiol-ene-methacrylate composites as dental restorative materials. Dent Mater. 2011 Mar;27(3):267-72. https://doi.org/10.1016/i.dental.2010.11.001

19. Maia AC, Mangabeira A, Vieira R, Neves AA, Lopes RT, Pires TM, et al. Experimental composites containing quaternary ammonium methacrylates reduce demineralization at enamel-restoration margins after cariogenic challenge. Dent Mater. 2019 Aug;35(8):e175-83. https://doi.org/10.1016/i.dental.2019.05.021

20. Rego GF, Vidal ML, Viana GM, Cabral LM, Schneider LF, Portela MB, et al. Antibiofilm properties of model composites containing quaternary ammonium methacrylates after surface texture modification. Dent Mater. 2017 Oct;33(10):1149-56. https://doi.org/10.1016/i.dental.2017.07.010

21. Vidal ML, Rego GF, Viana GM, Cabral LM, Souza JP, Silikas N, et al. Physical and chemical properties of model composites containing quaternary ammonium methacrylates. Dent Mater. 2018 Jan;34(1):143-51. https://doi.org/10.1016/j.dental.2017.09.020

22. Gosavi SS, Gosavi SY, Alla RK. Local and systemic effects of unpolymerised monomers. Dent Res J (Isfahan). 2010;7(2):82-7.

23. Soares AS, Scelza MZ, Spoladore J, Gallito MA, Oliveira F, Moraes RC, et al. Comparison of primary human gingival fibroblasts from an older and a young donor on the evaluation of cytotoxicity of denture adhesives. J Appl Oral Sci. 2018;26(0):e20160594 https://doi.org/10.1590/1678-7757-2016-0594

24. Nibali L, Fedele S, D’Aiuto F, Donos N. Interleukin-6 in oral diseases: a review. Oral Dis. 2012 Apr;18(3):236-43. https://doi.org/10.1111/j.1601-0825.2011.01867.x 